\title{
GYRE: A new open-source stellar oscillation code
}

\author{
Rich Townsend ${ }^{1} \dagger$, Seth Teitler ${ }^{1}$ and Bill Paxton ${ }^{2}$ \\ ${ }^{1}$ Department of Astronomy, University of Wisconsin-Madison, Madison, WI 53706, USA \\ ${ }^{2}$ Kavli Institute for Theoretical Physics, University of California \\ Santa Barbara, CA 93106, USA
}

\begin{abstract}
We introduce GYRE, a new open-source stellar oscillation code which solves the adiabatic/non-adiabatic pulsation equations using a novel Magnus Multiple Shooting (MMS) numerical scheme. The code has a global error scaling of up to 6th order in the grid spacing, and can therefore achieve high accuracy with few grid points. It is moreover robust and efficiently makes use of multiple processor cores and/or nodes. We present an example calculation using GYRE, and discuss recent work to integrate GYRE into the asteroseismic optimization module of the MESA stellar evolution code.
\end{abstract}

Keywords. methods: numerical, stars: evolution, stars: interiors, stars: oscillations

Interpreting the wealth of new observations provided by MOST, CoRoT and Kepler requires the theorist's analog to the telescope: a stellar oscillation code which calculates the eigenfrequency spectrum of an arbitrary input stellar model. Comparing a calculated spectrum against a measured one provides a concrete metric for evaluating a model, and therefore constitutes the bread and butter of quantitative asteroseismology.

There's no shortage of oscillation codes available to the community; the nine codes reviewed in Moya et al. (2008) are likely only a fraction of those being used on a day-today basis. However, automated asteroseismic optimization tools such as AMP (Metcalfe et al. 2009) and MESA (Paxton et al. 2013) are placing ever-increasing demands on these codes. A code will typically be executed hundreds or thousands of times during an optimization run, and must therefore make efficient use of available computational resources such as multi-processor hardware. The code must be robust, running and producing sensible output without manual intervention. The code must have an accuracy that matches or exceeds the frequency precision now achievable by satellite missions. Finally, it is preferable that the code address the various physical processes that inevitably complicate calculations, such as non-adiabaticity, rotation, and magnetic fields.

Currently, there are no publicly available oscillation codes which address all of these requirements. This motivated us to develop another code, 'GYRE', which is built on a novel Magnus Multiple Shooting (MMS) scheme for solving both adiabatic and non-adiabatic pulsation problems. GYRE and the MMS scheme are described in detail in a forthcoming paper (Townsend \& Teitler 2013). The code is written in standard-conforming Fortran 2008 with a modular architecture that allows straightforward extension to handle more complicated problems. To leverage multiple processor cores and/or cluster nodes, the code is parallelized using a combination of OpenMP and MPI.

As an illustration of GYRE in action, Fig. 1 presents results from a simulation exploring how the dipole-mode oscillation frequencies of a $1.5 M_{\odot}$ MESA stellar model change as the star evolves through the so-called red bump phase. (This phase occurs when $\mathrm{H}$ burning shell reaches the composition discontinuity left by the convective envelope after

\section{$\dagger$ email: townsend@astro.wisc.edu}




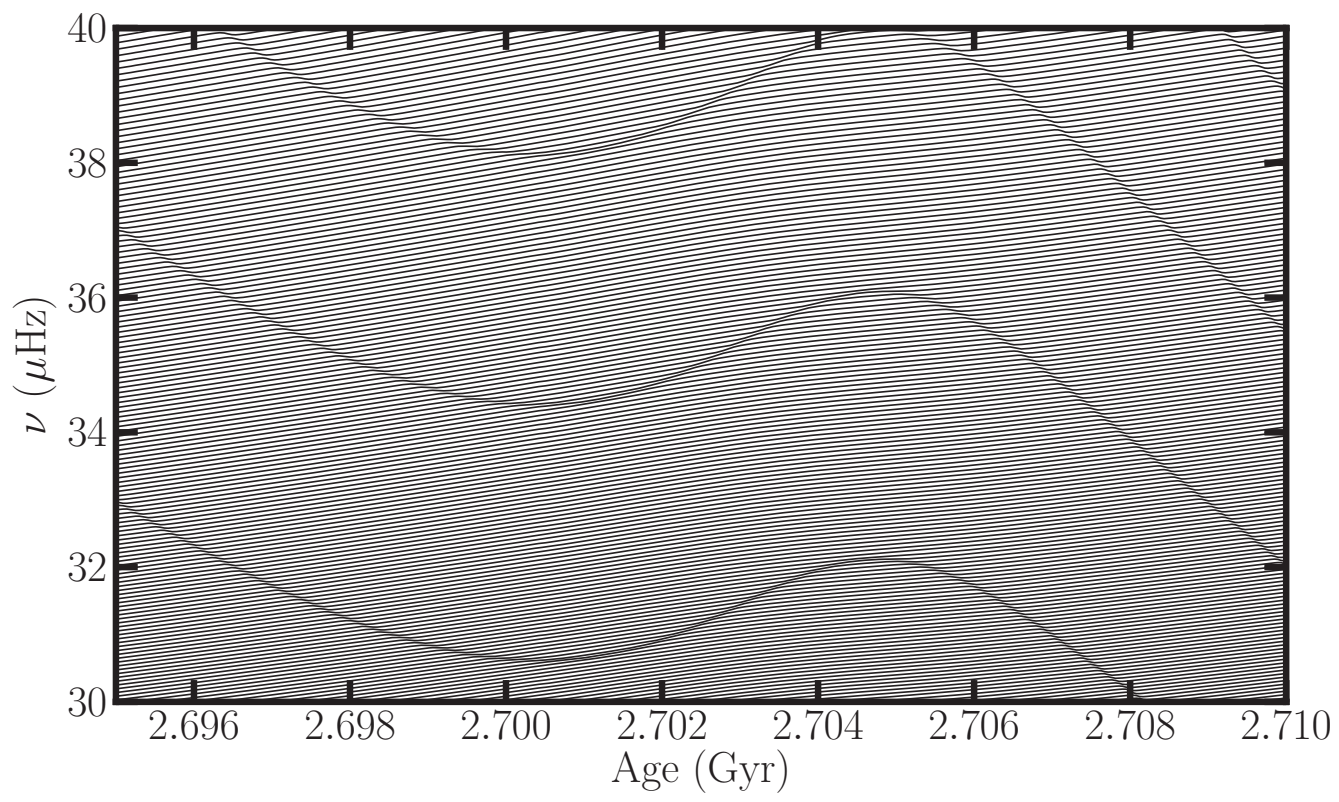

Figure 1. Dipole-mode linear eigenfrequencies of a 1.5 $M_{\odot}$ MESA model, plotted as a function of stellar age during evolution through the RGB bump phase. Coupling between envelope $\mathrm{p}$ modes and core g modes is revealed in the avoided crossings; the reversal in the time evolution of the crossing frequencies, between 2.700 and $2.705 \mathrm{Gyr}$, arises from the temporary contraction of the stellar envelope during the bump phase.

first dredge-up, causing a temporary reversal in the star's luminosity growth.) The figure reveals that the effects of bump passage can clearly be seen in the avoided crossings which arise from coupling between core g modes and envelope p modes: as the envelope contracts during the bump phase, the frequencies of the avoided crossings correspondingly increase.

Since revision 5232 MESA includes GYRE as one of the oscillation codes underpinning its asteroseismic optimization module (the other, currently, is ADIPLS). Communication between MESA and GYRE is accomplished through a simple application programming interface: MESA passes a model to GYRE, which then returns a list of modes having eigenfrequencies in a given range.

GYRE is open for use and distribution under the GNU General Public License; our hope is that a community of practice will arise around the code, bringing together users and developers to shape the code's future evolution in ways that best serve the field and its participants. Source code, documentation and other materials can be found at http://www. astro.wisc.edu/ ${ }^{\sim}$ townsend/gyre/.

\section{Acknowledgements}

We acknowledge support from NSF awards AST-0908688 and AST-0904607 and NASA award NNX12AC72G.

\section{References}

Metcalfe, T. S., Creevey, O. L., \& Christensen-Dalsgaard, J. 2009, ApJ, 699, 373

Moya, A., Christensen-Dalsgaard, J., Charpinet, S., et al. 2008, Ap\&SSS, 316, 231

Paxton, B., Cantiello, M., Arras, P., et al. 2013, ApJS, 208, 4

Townsend, R. H. D. \& Teitler, S. A. 2013, MNRAS, 435, 3406 